\title{
Denervation-Induced Changes in Cell Proliferation in the Rat Molar After Wounding
}

\author{
DANIEL J. CHIEGO, JR., ROBERT M. KLEIN, JAMES K. AVERY, AND IRIS M. GRUHL \\ Department of Oral Biology, University of Michigan, School of Dentistry, Ann Arbor, \\ Michigan 48109 (D.J.C., I.M.G., J.K.A.); Department of Anatomy/Division of Cell Biology, \\ University of Kansas, College of Health Sciences and Hospital, Kansas City, Kansas 66103
}

(R.M.K.)

\begin{abstract}
The dental pulp has the capacity to initiate and maintain repair after trauma. The purpose of the present study was to quantitatively analyze the role of the peripheral nervous system in regulation of pulpal cell proliferation in response to wounding. Six groups of ten rats were used in these studies. There was one baseline group (wounded, but innervation intact) and five resection groups. The resection groups included rats with unilateral superior cervical ganglionectomy (SCG), unilateral inferior alveolar nerve resection (IAN), unilateral chorda tympani (CT) resection, IAN + SCG, or a complete unilateral nerve resection (IAN + SCG + CT). One millimeter of enamel and dentin was removed from the first mandibular molar on the experimental (resected) side. Therefore, each rat had an experimental and control molar. Rats were killed at various intervals from day 0 to day 15 after wounding and received $0.5 \mu \mathrm{Ci} / \mathrm{g}$ b.wt. ${ }^{3} \mathrm{H}$-thymidine 1 hour before death. For the baseline (innervation intact) data a peak in ${ }^{3} \mathrm{H}$-thymidine incorporation occurred at 5 days after wounding. In the resected groups, there was a general increase in the number of labeled cells at the zero time point, and a suppression of the 5 -day peak with a delay in the proliferative response to wounding. The SCG + IAN-resected group maintained the lowest number of labeled cells throughout the entire experimental period compared to the experimental baseline data and the two controls. At the initial and termination points the SCG + IAN-resected groups demonstrated the highest number of labeled cells. The baseline data indicate that the maximal response to wounding occurs at day 5 . Denervation results in a delay of the cell proliferative response to wounding with the most dramatic delay occurring in the IAN + SCG-resection group. The results indicate that the autonomic and sensory components of the peripheral nervous system may interact in regulating the proliferative response of pulpal cells to wounding.
\end{abstract}

The capacity of the dental pulp to repair itself after wounding is well known, although how this response is mediated and regulated is still equivocal. Neuroanatomical tracing techniques that use various tritiated amino acids, horseradish peroxidase, and neurohistochemistry have shown that the dental pulp is densely innervated especially in the coronal and pulp horn regions (Avery et al., 1980). The pulpal innervation consists of A-delta and C-fibers carrying the modality of pain and subserving autonomic functions. It has been suggested by some investigators that the somatic and autonomic innervation of the tooth pulp may be involved in other functions exclusive of pain perception and vascular control (Byers, 1977; Piminidis and Hinds, 1977; Chiego et al., 1983). The auxilliary functions of the nervous system in regulation of growth, maintenance, and repair of specific target organs and cells is known as neurotrophism (Singer, 1952). Recently autonomic and sensory neural influences on the regulation of protein synthesis have been demonstrated in alveolar bone (Chiego and Singh, 1981; Singh et al., 1982).
The literature is equivocal regarding the effects of denervation on the development and formation of dental tissues as well as the eruption of teeth. Taylor and Butcher (1951) demonstrated that eruption rate of the continuously erupting rodent incisor was increased after resection of the inferior alveolar nerve (IAN). This was also demonstrated by Brown et al., (1961) and Devoto et al., (1966), although Torneck et al. (1972) could not corroborate the results of Taylor and Butcher (1951). Chiego et al. (1981) reported that the IAN resection produced an altered pattern of cellular proliferation in the mouse incisor, while chemical sympathectomy had only a minor effect on the progenitive compartments of the mouse (Klein et al., 1981).

In view of the above information, it was hypothesized that a component of the nervous system regulates specific cellular and/or subcellular functions including cellular proliferation, differentiation, and protein synthesis. The purpose of this study was to quantitatively analyze

Received April 17, 1985; accepted October 18, 1985. 
and compare the proliferative response of the dental pulp, after wounding, in intact rats and in rats with sensory and/or autonomic surgical denervation.

\section{MATERIALS AND METHODS}

Two groups of adult Sprague-Dawley rats (150-165 g) were maintained on a 12-hour light-dark cycle (lights on 0600 to 1800 hours EST). Sixty rats were included in group A (baseline data-wounded, but innervation intact) and divided into six subgroups: 0 day 6 hours, 0 day 7 hours, 0 day 8 hours, 5 days, 10 days, and 15 days. Group B consisted of 100 rats divided into five subgroups which had the inferior alveolar nerve (IAN), superior cervical ganglion (SCG), chorda tympani (CT), SCG + IAN or all three neural components resected on the right side 7 days prior to tooth wounding and were killed on days $0,5,10$, or 15 posttooth wounding. All rats had $1 \mathrm{~mm}$ of enamel and dentin removed from the occlusal surface of the right first mandibular molar using a $\$ 557$ carbide crosscut fissure bur and an ultraspeed rotary handpiece cooled with an air-water spray on day 0 at time 0 . The untreated contralateral molar was used as the control. Both surgical and tooth-wounding procedures were completed between 0800 and 1000 hours on the appropriate days of the experiments. Rats were injected with $0.5 \mu \mathrm{Ci} / \mathrm{g}$ b.wt. ${ }^{3} \mathrm{H}$-thymidine $(6.7 \mathrm{Ci} / \mathrm{mmole}$, New England Nuclear Co., Boston, MA.) i.p. 1 hour prior to death at the appropriate time interval. The mandibles were removed and placed in $10 \%$ phosphate-buffered formalin overnight, scraped free of soft tissue, and decalcified in EDTA. The mandibles were subsequently dehydrated in a graded series of alcohols, embedded in paraffin, sectioned at $5 \mu \mathrm{m}$, and placed on glass slides. The slides were then dipped in Kodak NTB-2 emulsion and exposed at $4{ }^{\circ} \mathrm{C}$ for 14 days. After developing and fixing, the autoradiograms were stained with Harris's hematoxylin and eosin and coverslipped. In a preliminary experiment, it was found that in the baseline (wounded, innervation intact) group approximately $94 \%$ of the labeled pulpal nuclei were identified as fibroblastlike cells and $6 \%$ endothelial cells. The criterion for a labeled cell was defined as the presence of four or more autoradiographic grains over the nucleus. In the present studies labeled pulpal cells were counted throughout the coronal pulp and pulp horns for each tooth and for all time periods and expressed as labeled cells/section. Five adjacent sections from the midsagittal plane were used in this study. Differences in pulpal areas of these sections were less than $5 \%$ for each tooth as determined by using a Zeiss Interactive Digital Analysis System (ZIDAS). Results from control and experimental teeth were statistically compared by using an unpaired t-test. Significant differences between groups were established at $\mathrm{P}<.05$.

\section{RESULTS}

Figure 1 graphically depicts the baseline (innervation intact) data. The experimental (wounded) molars in the baseline group demonstrated a distinct peak at 5 days as compared to the contralateral controls (significantly different, $\mathrm{P}<.05$ ) after which there was a decrease in the number of labeled pulpal cells throughout the 10 . day period to the final interval at 15 days postwounding (Fig. 1, baseline). In all of the neural resection experiments there are bilateral effects of unilateral denerva- tion which are evident when one compares either control (contralateral) or experimental (ipsilateral) molars to the baseline (innervation intact) data at the corresponding time intervals. For example, at 0 days the CT and SCG + IAN resections are significantly different from the baseline experimentals $(\mathrm{P}<.05)$. Effects of denervation on the contralateral tooth have been demonstrated and discussed previously (Devoto et al., 1966; Chiego et al., 1981).

Figure 1 (IAN) graphically compares the number of labeled pulpal cells in control and IAN-resected molars. Pulpal cells from control and experimental molars show a similar pattern through 10 days followed by an increase at 15 days. Compared to the baseline data there is an apparent delay in the pulpal response to wounding.

Figure 1 (SCG) is a graphical comparison of data from rats with resected superior cervical ganglia (SCG). Removal of the SCG results in the loss of the 5-day peak in ${ }^{3} \mathrm{H}$-thymidine labeling. Although the number of labeled pulpal cells was originally higher than the baseline there was, however, a large increase in the number of labeled cells at the 15-day time period. Significant differences (P $<.05$ ) between contralateral controls and experimentals occurred only at day 10 .

Figure 1 (CT) shows the effects of chorda tympani (CT) resection on control and experimental molars. From 0 to 5 days the CT-resected groups show a gradual decline while the baseline reaches maximal labeling. The pulpal cells in the control molar have a higher number of labeled cells at 0 days, but both groups show a similar pattern at 5,10 , and 15 days.

Figure 1 (SCG + IAN) represents the results of the SCG + IAN-resected data for control and experimental molars. Experimental rats with resected SCG + IAN maintained the lowest number of labeled cells throughout the entire study as compared to the baseline experimental data. High absolute labeling values were obtained at the 0-day point for the SCG + IAN controls. Significant differences $(\mathrm{P}<.05)$ between contralateral controls and experimentals were found in this group only at day 0 .

Figure 1 (complete) shows the results after complete $(\mathrm{SCG}+\mathrm{IAN}+\mathrm{CT})$ resection for control and experimental molars. At the initial time point of 0 day, the experimental group demonstrated more than double the labeling of the baseline controls. In addition, the initial time period for the complete resection groups represented the peak of ${ }^{3} \mathrm{H}$-thymidine-labeled cells in marked contrast to the baseline, which peaked at 5 days after the initial wounding.

\section{DISCUSSION}

The results of the baseline study indicate that when an intact innervation is present events involved in initiation of pulpal proliferation in the rat begin soon after the initial tooth trauma is induced and peak at 5 days after injury. Reports by other investigators have shown that cellular proliferation involved in repair processes in a number of experimental systems such as a fracture healing (Tonna and Cronkite, 1961), tape stripping of the epidermis (Hennigs and Elgjo, 1970), and regeneration of amphibian limbs (Tassava et al., 1974), is initiated rapidly after the tissue trauma. Under normal conditions pulpal cells are capable of cell proliferation 


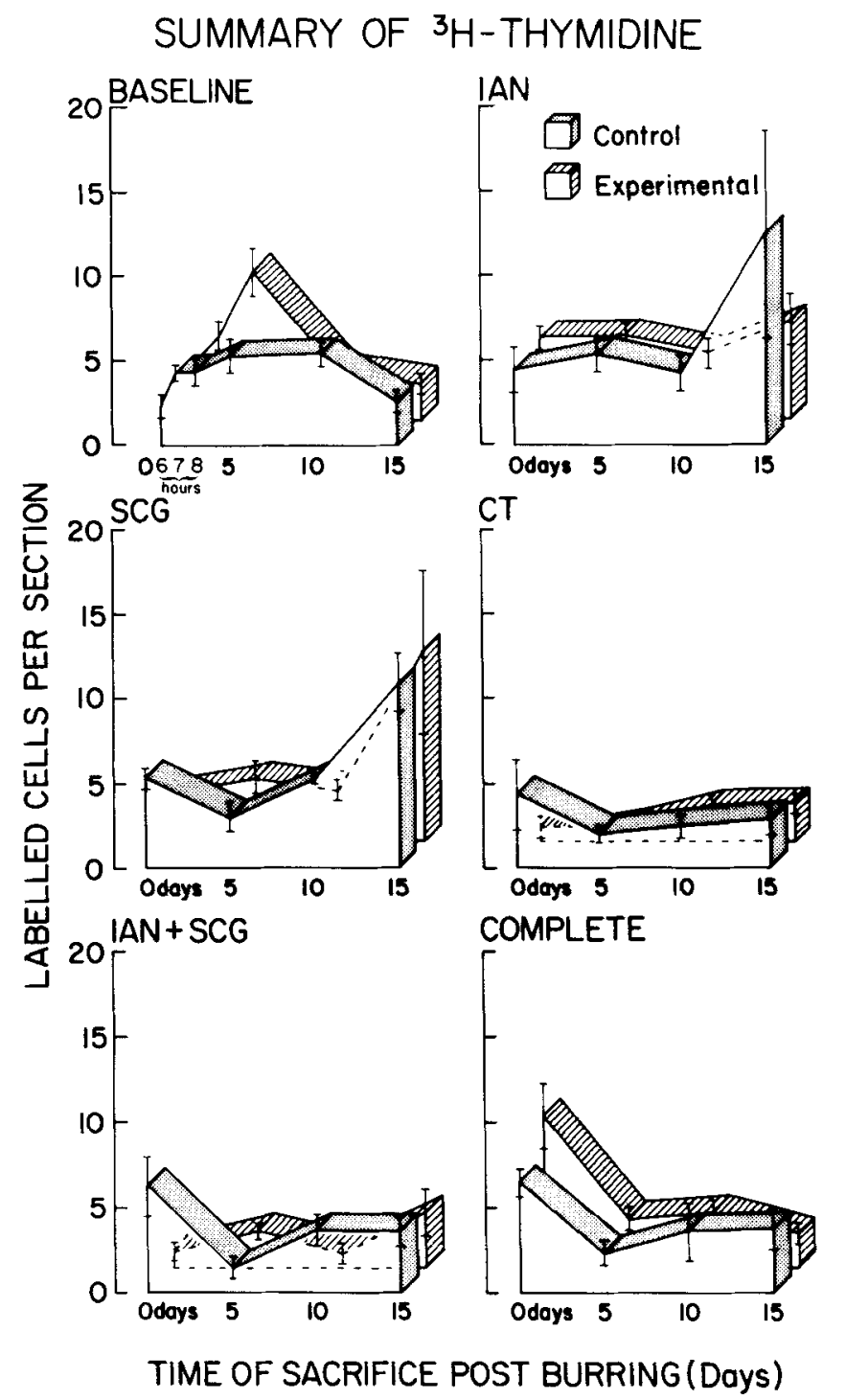

Fig. 1. The baseline data represent control vs. experimental (wounded) with an intact innervation. Standard errors of the means are delineated at each time interval. In each of the figures control (contralateral) and experimental (ipsilateral) data are graphically de-

and a slow rate of turnover (Hoffman and Gillette, 1960; Robins, 1967; Pinzon et al., 1970). Cotton (1968) found minimal ${ }^{3} \mathrm{H}$-thymidine labeling after shallow cavity preparation and concluded that the adult rat molar pulp consists of a stable cell population. However, there have been few quantitative studies of pulpal response to injury. Feit et al. (1970) analyzed ${ }^{3} \mathrm{H}$-thymidine incorporation into the pulp of rat incisors which were damaged by induction of narcosis with chloral hydrate. These authors found the highest density of labeled pulpal cells on the fourth day after injury, but found no labeled matured odontoblasts. Similar findings were demonstrated during dentinal bridge repair in rhesus monkeys with a peak in ${ }^{3} \mathrm{H}$-thymidine labeled pulpal cells occurring between days 4 and 6 after pulpal exposure followed by cavity preparation and restoration (Fitzgerald, 1979). picted following the specific denervation procedure. IAN, unilateral alveolar nerve resection; SCG, unilateral superior cervical ganglionectomy; CT, unilateral chorda tympani resection.

The present studies confirm that relatively quiescent pulpal cells are stimulated to enter the cell division cycle by wounding. Studies by Yamamura et al. (1980) have demonstrated that pulpotomy results in the dedifferentiation of mesenchymal cells which revert to undifferentiated pluripotential cells. The data of Yamamura et al. (1980) corroborate the results of Sveen and Hawes (1968), who demonstrated that tooth grinding stimulated mitotic activity of undifferentiated pulpal cells. These cells appear to enter the cell cycle before undergoing "redifferentiation" into odontoblasts (Yamamura et al., 1980).

There are a number of similarities between the repair processes which occur in the molar after wounding and the regenerating amphibian limb. In both cases mesenchymal-type cells are recruited by local dedifferentiation and enter the cell cycle (Hay and Fischman, 1961; Ya- 
mamura et al., 1980; Kintner and Brockes, 1984). In addition, both limb regeneration and tooth repair involve early sustained cell proliferation of newly dedifferentiated cells (Tassava and McCullough, 1978). Recent continuous labeling studies suggest that punctuated cell cycling occurs in the regenerating amphibian limb (Tomlinson et al., 1985). These authors hypothesize that the punctuation occurs in the $G_{1}$ phase of the cell cycle wherein cells may enter a "transiently quiescent" position. In the regenerating limb, injury and nerves are important factors in determining the capability of the limb to regenerate by influencing the nature of the cycling and noncycling compartments. The injury is the stimulus for entry of dedifferentiated cells into the cell cycle while the nerves influence the progression of these dedifferentiated cells through the cell cycle (Tomlinson et al., 1985).

In the present experiments, comparison of the baseline data to the results after neural resection indicates that denervation of rat molars results in inhibition of the proliferative capacity of the pulp. The inhibitory role of the nervous system in regulation of pulpal cell proliferation may be analagous to the regenerative response of denervated amphibian limbs. In the regenerating amphibian limb Singer (1952) has suggested that the role of nerves is to create new events by increasing the rate of mitotic activity and not by the introduction of new and unique mechanisms. The nerves primarily influence the $G_{1}$ phase of the cell cycle during limb regeneration (Globus, 1978; Tassava et al, 1983, Tomlinson et al., 1984) although following denervation some cells enter the cell cycle, but do not undergo mitosis and become blocked in the $\mathrm{G}_{2}$ phase of the cell cycle (Tassava and McCullough, 1978). In the present study, denervation of the autonomic or sensory components to the teeth may alter the passage of odontoblasts through the cell cycle and the entry or reentry of cells into the proliferating compartment as occurs in the regenerating amphibian limb (Tomlinson et al., 1985). However, continuous ${ }^{3} \mathrm{H}$ thymidine-labeling and cell cycle studies will be required to answer these questions.

In the present studies, the cell proliferative pattern varies following different denervation procedures. However, there is a generalized delay or inhibition of thymidine incorporation on the experimental side in most groups. Previous ${ }^{3} \mathrm{H}$-thymidine-labeling index studies of the proliferative compartments of the continuously erupting mouse incisor at 15,30 , and 60 days after IAN denervation indicate as complex pattern of cell proliferative alterations (Chiego et al., 1981). Labeling index of pulpal fibroblasts was significantly decreased compared to sham controls at 15,30 , and 60 days following IAN resection. Other cellular compartments demonstrated alterations in labeling index during the early time periods followed by a return toward control levels. This pattern is indicative of an intrinsic compensatory response to the absence of sensory innervation by the continuously erupting rat incisor. In this respect, the continuously growing rodent incisor may be similar to other continuously renewing systems, such as the small intestinal epithelium, which appear to adapt to the $a b-$ sence of innervation (Musso et al., 1975). In contrast to IAN resection, chemical sympathectomy or guanethidine treatment does not appear to affect eruption rate (Moxham, 1979) or the labeling index of most progeni- tive compartments in the continuously erupting rodent incisor (Klein et al., 1981). However, these data were obtained by using the continuously erupting rodent incisor as a model system, which is not adequate for comparison to noncontinuously renewing teeth such as molars.

In the current experiments, the complete resections (IAN + SCG + CT) demonstrated a different ${ }^{3} \mathrm{H}$-thymidine incorporation pattern on the experimental side than occurred after the individual denervation procedures or the IAN + SCG resections. In previous studies IAN or IAN + SCG denervation has been shown to affect the pattern and quality of dentin formation to a similar degree (Avery et al., 1974). However, a recent study in our laboratory demonstrated an increase in the incorporation of ${ }^{3} \mathrm{H}$-fucose into odontoblasts after IAN and/or SCG resections (Chiego et al., 1983). Absolute incorporation of ${ }^{3} \mathrm{H}$-fucose was significantly increased by $48 \%$ after SCG resection, by $24 \%$ after IAN resection, and by $14 \%$ after sensory and sympathetic denervation. In the present study, IAN + SCG resection in experimental rats resulted in the lowest number of labeled cells throughout the entire study as compared to the experimental baseline data. In contrast, complete resection resulted in an early peak in ${ }^{3} \mathrm{H}$-thymidine incorporation at the initial time interval indicating an increased recruitment of cells into the cell cycle in this group. Resection of the IAN + SCG resulted in a similar early peak in the contralateral (nonwounded) molar tooth. These results combined with the data from the ${ }^{3} \mathrm{H}$-fucose study indicate a possible neural interaction between the autonomic and sensory innervation of the dental pulp.

Although the mechanisms involved in the neural regulation of cell proliferation in the tooth pulp are unknown, cyclic nucleotides (Davidovitch et al., 1977), prostaglandins (Dekel et al., 1981), and regional acceleratory phenomena (Frost, 1983) have been implicated in the regulation of hard tissue response to injury. Increased prostaglandin production has been demonstrated in rabbits after tibial fractures and may be related to proliferation of osteogenic cells (Dekel et al., 1981). The cyclic nucleotides are involved in the mediation of neural effects in other organ systems. Electrical stimulation of both branches of the autonomic nervous system results in alteration of cyclic nucleotide concentration within the parotid gland (Muir and Templeton, 1976; Templeton et al., 1977). Altered cyclic nucleotide levels may also be associated with stimulation of proliferation and differentiation in periodontal ligament fibroblasts during orthodontic tooth movement (Davidovitch, 1978; Roberts and Chase, 1981). While cyclic nucelotides may be involved in the intracellular mediation of neural influences, Ruch et al. (1982) have suggested that epigenetic controls are required for the progressive realization of the genetically determined phenotype of the odontoblast. Odontoblasts appear to undergo progressive maturation until their terminal differentiation, which can be delayed by lengthening the $G_{1}$ phase of the cell cycle, allowing for transcriptional changes. Finally during the last cell division the preodontoblasts align perpendicular to the basement membrane and begin matrix secretion. This last step, polarization of the odontoblast, has been postulated to be modulated by cell-membrane-bound receptors and is followed by an increase in intracellular cyclic AMP 
(Ruch et al., 1982). Future studies in this laboratory will attempt to determine the effects of denervation on cyclic nucleotide levels in odontoblasts and other cell types in the tooth and the role of various neural components in odontoblastic function and differentiation.

\section{ACKNOWLEDGMENTS}

This work was supported by N.I.H. grant DE05636 from the National Institute of Dental Research. The authors thank Martha O'Donnell for her careful typing of the manuscript.

\section{LITERATURE CITED}

Avery, J.K., C.F. Cox, and D.J. Chiego, Jr. (1980) Presence and location of adrenergic nerve endings in the dental pulps of mouse molars. Anat. Rec., 198:59-71.

Avery, J.K., C.F. Cox, and R.E. Corpron (1974) The effects of combined nerve resection and cavity preparation and restoration on response dentin formation in rabbit incisors. Arch. Oral Biol., 19:539-548.

Brown, G.N., S.R. Kupfer, and C.G. Darlington (1961) Effects of inferior alveolar nerve severance on the eruption rate of the mandibular incisor in the ten day old Wistar albino rat. Oral Surg., Oral Med., Oral Pathol., 14:1227-1255.

Butcher, E.O., and A.C. Taylor (1951) The effects of denervation and ischemia upon the teeth of the monkey. J. Dent. Res., 30:265-275.

Byers, M.R. (1977) Fine structure of trigeminal receptors in rat molars. In: Pain in the Trigeminal Region, D.J. Anderson and B. Matthews, eds. Elsevier/North Holland Biomedical Press, N.Y., pp. 13-24.

Chiego, D.J. Jr., R.M. Klein, and J.K. Avery (1981) Tritiated thymidine autoradiographic study of the effects of inferior alveolar nerve resection on the proliferative compartments of the mouse incisor formative tissues. Arch. Oral Biol., 26:83-89.

Chiego, D.J. Jr., M.A. Fisher, J.K. Avery, and R.M. Klein (1983) Effects of denervation on ${ }^{3} \mathrm{H}$-fucose incorporation by odontoblasts in the mouse incisor. Cell Tissue Res., 230:197-203.

Chiego, D.J. Jr., and I.J. Singh (1981) Evaluation of the effects of sensory denervation on osteoblasts by ${ }^{3} \mathrm{H}$-proline autoradiography. Cell Tissue Res., 217:569-576.

Cotton, W.R. (1968) Pulp response to cavity preparation as studied by the method of thymidine- $\mathrm{H}^{3}$ autoradiography In: Biology of the Dental Pulp Organ. S.B. Finn, ed. Symposium, Univ. of Alabama Press, University, Alabama, pp. 69-101.

Davidovitch, Z., P.C. Montgomery, R.W. Yost, and J.L. Shanfeld (1977) Immuno-histochemical localization of cyclic nucleotides in the periodontium: Mechanically-stressed cells in vivo. Anat. Rec., 192:351361 .

Dekel, S., G. Lenthall, and M.J.O. Francis (1981) Release of prostaglandins from bone and muscle after tibial fracture. J. Bone Joint Surg., 63-B:185-189.

Devoto, R.H., N.H. Aries, and B.M. Perrotto (1966) Growth of rats lower incisor teeth after unilateral section of alveolar nerve. J. Dent. Res., 45;1078-1082.

Feit, J., M. Metelovia, and Z. Sindelka (1970) Incorporation of ${ }^{3} \mathrm{H}$ thymidine into damaged pulp of rat incisors. J. Dent. Res., 49:783786.

Fitzgerald, M. (1979) Cellular mechanics of dental bridge repair using ${ }^{3} \mathrm{H}$-thymidine. J. Dent. Res., 58:2198-2206.

Frost, H.M. (1983) The regional acceleratory phenomenon: A review, Henry Ford Hosp. Med. J., 31:3-9.

Globus, M. (1978) Neurotrophic contribution to a proposed tripartite control of the mitotic cycle in the regeneration blastema of the newt, Notophthalmus viridescens. Am. Zool, 18:855-868.

Hay, E.D., and D.A. Fischman (1961) Origin of the blastema in regenerating limbs of the newt Triturus viridescens. An autoradio graphic study using tritiated thymidine to follow cell proliferation and migration. Dev. Biol., 3:26-59.

Hennings, H., and K. Elgjo (1970) Epidermal regeneration after cellophane tape stripping of hairless mouse skin. Cell Tissue Kinet., 3:243-252.

Hoffman, R.L., and R. Gillette (1962) Mitotic patterns in pulpal and periodontal tissue of developing teeth. J. Dent. Res., 40:76-87.

Kintner, C.R., and J.P. Brockes (1984) Monoclonal antibodies identify blastema cells derived from dedifferentiating muscle in newt limb regeneration. Nature, 308:67-69.

Klein, R.M., D.J. Chiego, Jr., and J.K. Avery (1981) Effects of chemical sympathectomy on cell prolifertion in the progenitive compart ments of the neonatal mouse incisor. Arch. Oral Biol., 26:319-325.

Moxham, B.J. (1979) The effects of some vaso-active drugs on the eruption of the rabbit mandibular incisor. Arch. Oral Biol. 24:681688.

Muir, T.C., and D. Templeton (1976) The role of cyclic $3^{\prime}, 5^{\prime}$-adenosine monophosphate (cyclic AMP) in the ability of sympathetic nerve stimulation to enhance growth and secretion in rat salivary glands in vivo. J. Physiol. (Lond.), 259:47-61.

Musso, F., J.-J. Lachat, A.R. Cruz, and R.P. Goncalves (1975) Effect of denervation on the mitotic index of the intestinasl epithelium of the rat. Cell Tissue Res., 163:395-402.

Piminidis, M.Z., and J.W. Hinds (1977) An autoradiographic study of the sensory innervation of teeth, dental pulp and periodontium. J. Dent. Res., 56:835-840.

Pinzon, R.D., P.D. Toto, and J.J. O'Malley (1966) Kinetics of rat molar pulp cells at various ages. J. Dent. Res., 45:934-938.

Roberts, W.E., and D.C. Chase (1981) Kinetics of cell proliferation and migration associated with orthodontically-induced osteogenesis. J. Dent. Res., 60:174-181.

Robins, M.W. (1967) The proliferation of pulp cells in rat incisors. Arch. Oral Biol., 12:487-501.

Ruch, J.V., H. Lesot, V. Karcher-Djuricic, J.M. Meyers, and M. Olive (1982) Facts and hypotheses concerning the control of odontoblast differentiation. Differentiation, 21:7-12.

Singer, M. (1952) The influence of the nerve in regeneration of the amphibian extremity. Q. Rev. Biol., 27:169-200.

Singh, I.J., M.S. Herskovits, D.J. Chiego, Jr., and R.M. Klein (1982) Modulation of osteoblastic activity by sensory and autonomic in nervation of bone. In: Factors and Mechanisms Influencing Bone Growth. A.D. Dixon and B.G. Sarnat, eds. Alan R. Liss, Inc., N.Y. pp. 535-551.

Sveen, O.B., and R.R. Hawes (1968) Differentiation of new odontoblasts and dentine bridge formation in rat molar teeth after tooth grinding. Arch. Oral Biol., 13:1399-1412.

Tassava, R.A., L.L. Bennett, and G.D. Zitnik (1974) DNA synthesis without mitosis in amputated denervated forelimbs of larval axolotls. J. Exp. Zool., 190:111-116.

Tassava, R.A., and W.D. MeCullough (1978) Neural control of cell cycle events in regenerating salamander limbs. Am. Zool., 18:843-854.

Tassava, R.A., D.P. Treece, and C.L. Olsen (1983) Effects of partial denervation on the newt blastema cell cycle. In: Limb Development and Regeneration. Part A. J.F. Fallon and I.A. Caplan, eds. Alan R. Liss, Inc., New York, pp. 537-545

Taylor, A.C., and E.O. Butcher (1951) The regulation of eruption rate in the incisor teeth of the white rat. J. Exp. Zool., 17:165-168.

Templeton, D., F.R. Butcher, C.J. Turner, T.C. Muir, and J.P. Durham (1977) Effect of electrical stimulation of the autonomic nerves on the levels and synthesis of cylclic nucleotides in the rat salivary glands: Relationship to enhanced growth. J. Cyclic Nucleotide Res. 3:107-118.

Tomlinson, B.L., M. Globus, and S. Vethamany-Globus (1984) The blastema cell cycle in vitro and attempted restimulation of blas. tema cell cycling in denervated blastemata of the adult newt, Notophthalmus viridescens. J. Exp. Zool., 232:249-258.

Tomlinson, B.L., D.J. Goldhamer, P.M. Barger, and R.A. Tassava (1985) Punctuated cell cycling in the regeneration blastema of urodele amphibians. An hypothesis. Differentiation, 28:195-199.

Tonna, E.A., and E.P. Cronkite (1961) Cellular response to fracture studied with tritiated thymidine. J. Bone Joint Surg., 43-A:352362 .

Torneck, C.D., B. Harnett, T. Johnson, and T. Leung (1972) The effect of surgical denervation on dentin in the incisor of the rat. J. Dent. Res., 51:48-52.

Yamamura, T., M. Shimono, H. Koike, M. Terao, Y. Tanaka, Y. Sakal, T. Inoue, S. Yoshiki, T. Tachikawa, H. Kawahara, and O. Watanabe (1980) Differentiation and induction of undifferentiated mesenchymal cells in tooth and periodontal tissue during wound healing and regeneration. Bull. Tokyo Dent. Coll., 21:181-222. 